Conclusion To achieve digital change multiple enablers need to be undertaken to achieve change and the success is reflective of the multiple-pronged response.

\section{P-98 A MODERN VIEW OF HOSPICE DATA}

Fernando Torres. St. Cuthbert's Hospice, Durham, UK

\subsection{6/bmispcare-2019-HUKNC.121}

Background St. Cuthbert's Hospice manages their patients' records through SystmOne, a known patient record software. All the care services, in-patient and out-patient, are managed through the software, therefore it was possible to register outcome data and link it to a particular user, service, spell of care and phase of illness. This opened the path for developing an information system containing online-cloud interactive dashboards, all done with open source technologies, making it an affordable option. The system takes a patient-centred approach, which includes outcome scores per patient and their 'journey' across all services, having spells shared across the entire organisation and not by service, everything in one interface and accessible from any device with internet connection.

Methods The information system works with the data structure of SystmOne, allowing the inclusion of any validated outcome measurement, not only limited to IPOS. The system is made entirely with the $\mathrm{R}$ programming language, using the Rstudio ecosystem and is intended to centralise all the data that a hospice produces from every patient.

Conclusions Having a centralised information system allows hospices to see patients from every perspective, either if an in-patient or out-patient service was delivered. By having spells shared across all services it is possible to see sequentially how the patient has improved or deteriorated and how every department had an impact on the outcome. As most of the technology is open source, any hospice that currently uses SystmOne could make use of the system with little investment. Also, being able to see the data from any device connected to the internet can allow practitioners to access the patient's outcome even when they are performing a home visit.

\section{P-99 DRIVING BY THE DASHBOARD LIGHTS}

Duncan Fleming, John Sharman. Mountbatten, Isle of Wight, UK

10.1136/bmjspcare-2019-HUKNC.122

Background There was a need identified that data we had used and displayed historically didn't give a true picture of the complexity and diverse nature of the care and support we offered.

Aims

- To develop a dashboard as a single source of reporting for all different meetings, stakeholders and teams, from the Clinical Commissioning Group through to teams on the frontline;

- To have a consistent approach and method of reporting for all teams;

- To make sure all teams were represented;

- To highlight the importance of good recording.

Method When we moved to a new electronic patient record (EPR), this was an ideal opportunity to change the way we reported and demonstrated our work. A new role was recruited and an application called Power BI was used to analyse data from the EPR.

Results We found that the new dashboards promoted discussion in all the situations it was displayed and that staff engaged with data and recording at all levels of the organisation. Being able to see the data for individual teams has helped staff to understand the importance of recording accurately. Team leaders have fed back that they have a clear understanding of their caseloads and the complexity of patients referred to them using outcome measures.

The dashboard data acts as a single source of information for team and organisation reports, and sections of the dashboard have been used for delivering messages to the public. Having a dedicated member of staff has meant that changes can be made to the dashboard quickly as new data is requested.

Conclusion The development of our dashboards has allowed the organisation to give a clear picture of what we do. Feedback has been positive at all levels with engagement driving healthy discussion and improvements in practice.

\section{P-100 EVALUATION OF THE USE OF HEALTH INFORMATION EXCHANGE AT ST JOSEPH'S HOSPICE, LONDON}

${ }^{1,2}$ Samantha Edward. 'North London Hospice, London, UK; ${ }^{2}$ St Joseph's Hospice, London, UK

\subsection{6/bmjspcare-2019-HUKNC.123}

Background Shared care records is identified as one of the foundations to achieving the Ambitions for Palliative and End of Life Care (National Palliative and End of Life Care Partnership, 2015). Following many years of engagement with the IT Enabler Board in Hackney, St Joseph's Hospice gained access to the Health Information Exchange (HIE) in July 2017. The King's Fund in their review of successful digital change projects identified this link with the voluntary sector services provided at St Joseph's Hospice, as the first link of its kind (Maguire, Evans, Honeyman \& Omojomolo, 2018). The evaluation was undertaken after the first 1000 accesses.

Method During December 2018-January 2019, email questionnaires based on the benefits study (Redman et al.) were circulated to the 34 users. Follow-up reminder emails were sent. 12 respondents (seven nurses, five doctors) submitted between December 2018 and March 2019 (excluding the author). The completed questionnaires were evaluated.

Results The respondents identified that they used HIE to access a range of clinical information. Over $80 \%$ of the responding doctors and nurses used it to source information when taking a history. Nurses particularly reported this being a 'much easier' way to source information. $100 \%$ of nurses and $80 \%$ of doctors felt it had a 'positive' or 'very positive' effect on their working day.

Conclusion The benefits of access to the eLPR (east London Patient Record) have been suggested in this small sample of the first users of HIE in a hospice setting. The overwhelming weight of the responses regarding the benefits of access to information were positive. The results were limited by a low response rate. The findings do suggest that hospices could have considerable benefits with reference to access to information, reduction in administration and efficiency of gathering clinical information from other providers. The author recommends that hospices should explore the digital networks in 